\title{
REAL-TIME, COOPERATIVE ENTERPRISES: REQUIREMENTS AND SOLUTION APPROACHES
}

\author{
L. Monostori ${ }^{1,2}$, J. Váncza ${ }^{1,2}$, T. Kis ${ }^{1}$, B. Kádár ${ }^{1}$, Zs.J. Viharos ${ }^{1}$ \\ ${ }^{1}$ Computer and Automation Research Institute, \\ Hungarian Academy of Sciences, Budapest, Hungary \\ ${ }^{2}$ Department of Production Informatics, Management and Control, \\ Budapest University of Technology and Economics, Budapest, Hungary
}

\begin{abstract}
In addition of the four main R\&D directions, i.e., adaptive manufacturing, digital manufacturing, knowledge-based manufacturing, and networked manufacturing, emphasised by the European initiative Manufuture, two additional requirements, i.e., the real-timeness and cooperativeness of production structures are also outlined in the paper, which are of high and increasing importance. The paper highlights the main results of a national R\&D project in Hungary on Digital Enterprises, Production Networks, and the main concept of a new, just started, national research project VITAL: Real-time Cooperative Enterprises. Copyright (C) 2006 IFAC
\end{abstract}

Keywords: Digital Enterprises, Production Networks, Intelligent Manufacturing,

\section{INTRODUCTION}

Manufacturing systems of our days work in a fast changing environment full of uncertainties. Increasing complexity is another feature showing up in production processes and systems, furthermore, in enterprise structures as well (Wiendahl and Scholtissek, 1994). One of the recent areas of research is related to the globalization of production. Production networks (PNs) (Wiendahl and Lutz, 2002) are formed from independent companies collaborating by shared information, skills, resources, driven by the common goal of exploiting market opportunities.

The concept of the digital enterprise (Maropoulos, 2002), i.e., the mapping of the key processes of an enterprise to digital structures by means of information and communication technologies (ICT) gives a unique way of managing the above problems. By using recent advances of ICT, theoretically, all the important production-related information is available and manageable in a controlled, userdependent way (Monostori, et al., 2003).

However, the management, the optimal or near to optimal exploitation of this huge amount of information cannot be imagined without the effective application of the methods and tools of artificial intelligence (AI), sometimes, more specifically, machine learning (ML) techniques (Monostori, et al., 1996).

The detection of changes and uncertainties is one of the most important requirements of today's manufacturing. In (Monostori, 2003), the application of pattern recognition (PR) techniques, expert systems (ESs), artificial neural networks (ANNs), fuzzy systems (FSs) and hybrid AI techniques in manufacturing are outlined as consecutive elements of a process started two decades ago. Agent-based (holonic) systems are also highlighted as promising tools for managing complexity, changes and disturbances in production systems. The further integration of approaches is predicted.

\subsection{The Manufuture Initiative}

On December 1-2, 2003 the conference Manufuture was held in Milan, Italy, with the goal of calling the attention of the main stakeholders on the importance of manufacturing, the "general transformation of all resources to meet human needs" in the society. In the 
accompanying Working Document (N.N., 2003), five driving forces were identified for Manufuture:

- Increased research and technological development with longer term research-industry relationships and clearer support to industrial research.

- International cooperation in manufacturing research.

- The key role of education and training (attractiveness for the young, multi-disciplinarity, education - research - innovation, "training factories”).

- Stimulating operating environment for industrial innovation (innovating SMEs, intellectual property rights, Euro-patent, etc.).

- Increased competitiveness of European research (new funding instruments, increased networking to reduce fragmentation, long-term vision).

Four main directions were further emphasized at the Manufuture Workshop, July 1, 2004, Dortmund, Germany: adaptive manufacturing, digital manufacturing, knowledge-based manufacturing, networked manufacturing. In the coming space the above main research and development directions will be characterised in short, pointing on some important overview papers.

Adaptive manufacturing; In a landmark paper by J. Hatvany in 1983, Intelligent Manufacturing Systems (IMSs) were outlined as the next generation of manufacturing systems expected to solve, within certain limits, unprecedented, unforeseen problems on the basis of even incomplete and imprecise information (Hatvany, 1983).

Learning factories, enterprises in manufacturing must be conscious of their duality, i.e., the interdependence of their technical and human constituents. Though having concentrated on machine learning techniques for manufacturing in the extensive survey (Monostori, et al., 1996), the authors underlined that human and machine learning are equally essential for these learning enterprises. $\mathrm{K}$. Ueda and his co-workers address emergent synthesis methodologies for manufacturing applications and categorise the problems according to the incompleteness of information on the environment and/or the specification (Ueda, et al., 1998):

- Class l: Problem with complete description: if the information concerning the environment and specification are entirely given, then the problem is completely described. However, it is often difficult to find an optimal solution.

- Class II: Problem with incomplete environment description: the specification is complete, but the information on the environment is incomplete. Since the problem is not wholly described now, it is difficult to cope with the dynamic properties of the unknown environment.

- Class III: Problem with incomplete specification: not only the environment description but also the specification is incomplete. Problem solving, therefore, has to start with an ambiguous purpose, and the human interaction becomes significant.
The related survey (Ueda, et al., 2001) shows that the current status of studies related to emergent synthesis indicates most intensive research for Class II problems with increasing focus on Class III problems.

Digital manufacturing; Obviously, digital enterprise technologies (DET), i.e., "the collection of systems and methods for the digital modelling of the global product development and realization process in the context of life-cycle management" (Maropoulos, 2002), constitute one of the most promising approaches towards the future of manufacturing, i.e., "Manufuture". In the same paper, five main technical areas are outlined as cornerstones for realizing digital enterprises:

- distributed and collaborative design,

- process modelling and process planning,

- production equipment and factory modelling,

- digital to physical environment integrators,

- enterprise integration technologies.

Knowledge-based manufacturing; The application of knowledge-based techniques in manufacturing started several decades ago. For some details, let us refer to a survey from 1997 (Teti and Kumara, 1997). However, according to (N.N., 2004), novel systems do not only make use of knowledge to optimise specific production resources and processes, but also capture that knowledge and transfer it via knowledge platforms and competence networks to other areas where it can be employed to advantage. The knowledge from all fields of manufacturing has to be integrated - from manufacturing networks down to the individual components of manufacturing systems in order to enable companies to quickly respond to changes in a dynamic environment.

One of the first endeavours in the above direction is the establishment of the Network of Excellence (NoE) on Virtual Research Laboratory for a Knowledge Community in Production (http://www.vrl-kcip.org/) supported by the EU.

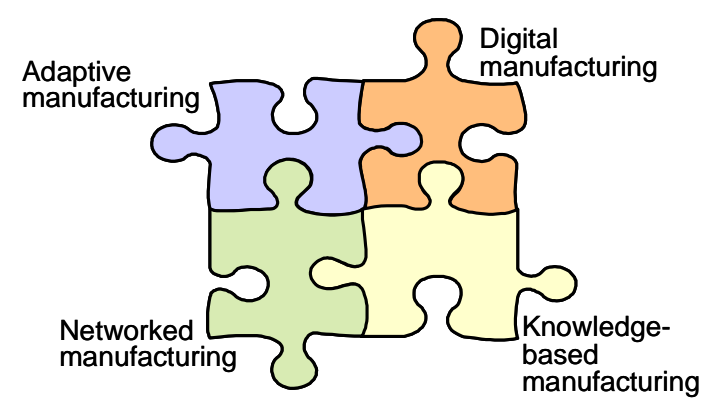

Fig. 1: Four important R\&D areas outlined in (N.N., 2004).

Networked manufacturing; A newly emerging area of research, representing a real challenge for the planning and management of production systems, is related to the paradigm of production networks. The determination of methods for the identification and verification of the manufacturing requirements of all parties in the network, as well as the specification of 
the necessary processes and ICT systems, will be central tasks for researchers in this field (N.N., 2004).

However, it must be pointed out that the four important areas shown in

Fig. 1 represent overlapping domains and can be considered in a holistic way only.

\subsection{Necessity of Real-Timeness and Cooperativeness}

At least two important requirements, i.e., the realtimeness and cooperativeness of the whole system, have to be added as issues of high and increasing importance. The first one refers to the ability of recognising and acting on internal and external changes and disturbances within the time frameworks required by the given level of the manufacturing-production hierarchy. The latter underlines the fact that the complex production structures - from machine tools, robots, etc. to production networks, including human beings involved - are more and more considered and built up as autonomous, but cooperative entities.

The fundamental aim of the paper is to illustrate how the above $R \& D$ directions outlined above are manifested in two large-scale national R\&D projects, i.e., one on Digital Enterprises, Production Networks, the other on Real-time, Cooperative Enterprises, by illustrating the results achieved and future research directions, as well.

\section{MAIN RESULTS OF THE NATIONAL PROJECTON DIGITAL ENTERPRISES, PRODUCTION NETWORKS}

The results presented here have been conceived and developed in the framework of a project run in Hungary on Digital Enterprises, Production Networks (Monostori, et al., 2003), (Monostori, et al., 2002).

The main intention of the partners was to develop solutions which are based on novel fundamental research, but, at the same time, applicable in the industry. The integrative endeavour of the partners was to make all the production-related information available and manageable in a controlled, userdependent way by the efficient use of information and communication technologies, i.e., to develop decision support systems, in order to help enterprises to cope with the problems of uncertainty and complexity, increase their efficiency, join in production networks and to improve the scope and quality of their customer relationship management.

The partners wanted to make progress in the following - partly overlapping - directions, project clusters:

- management and scheduling of large-scale projects,

- tele-presence and interactive multimedia,

- monitoring of complex production structures.
Because of the limited space only the main results of the first cluster is presented below

\subsection{Management and Scheduling of Large-Scale Projects}

Project-oriented production and capacity planner; The approach elaborated is applicable primarily in industries where complex, resource and cost intensive one-of-a-kind products are made on an engineering-to-order basis. Further on, production in a network was also considered where decisions on the allocation of tasks and the use of resources should concern both internal and external capacities; where the internal flow of materials should be synchronized with the incoming and outgoing flows (Wiendahl and Lutz, 2002). All this makes the problem of planning extremely hard to solve. Conversely, the complex situations call for efficient, robust decision support methods. Hence, there is a need for intuitive and flexible models and fast, reliable solution techniques that scale-up well also to large problem instances (Monostori, et al., 2003).

To handle this complexity the principle of aggregation was applied and certain details in the representation of products and orders, production processes, resource capacities, as well as of time were removed (Váncza, et al., 2004).

The basic idea is to model orders as projects (

Fig. 2) that compete for a number of limited-capacity resources (Márkus, et al., 2003). The method developed is based on a generalized version of the resource-constrained project scheduling problem and unifies the resource and the material flow oriented aspects of production and capacity planning (Kovács, et al., 2003).

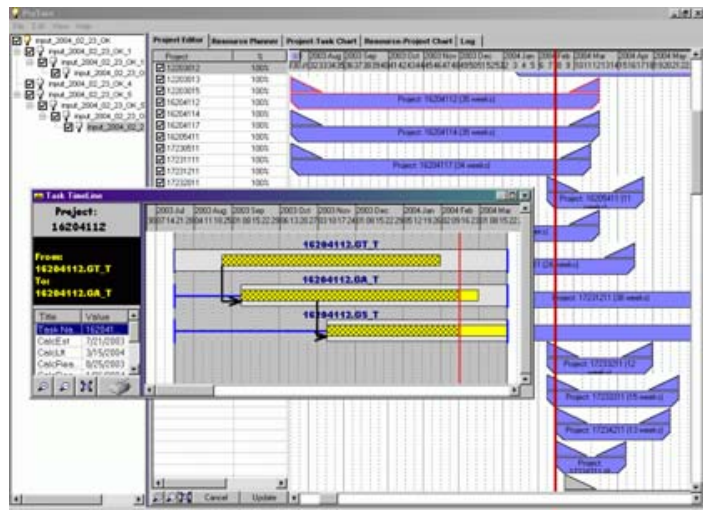

Fig. 2: Project view of production plans.

Constraints due to the limited capacities of resources (

Fig. 3) and precedence relations between the activities are prescribed. The classical model which assumes fixed activity durations and a constant rate of resource utilisation during the entire processing of every activity has been extended so that

- each activity can be executed with varying (even zero) intensity, 
- resources can be shared out among activities continuously.

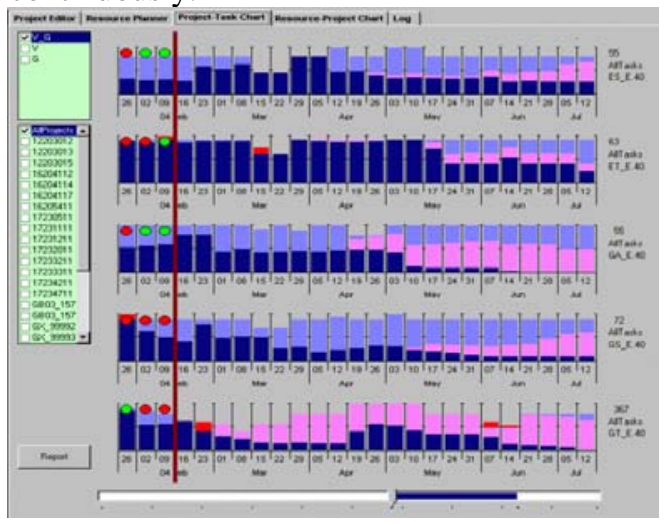

Fig. 3: Capacity view of production plans.

Having introduced variable-intensity tasks and continuous resources, the model can be solved by customized mathematical programming methods very efficiently (Kis, 2005). Hence, it can be applied in a dynamic setting when re-planning is initiated by unexpected changes. By using the system, what-if questions can be easily answered, as well.

Integrated production planner and scheduler; The solution developed within the project bridges the gap between the Enterprise Resource Planning (ERP) and Manufacturing Execution System (MES), by offering the necessary planning, scheduling and control functions (Fig. 4).

The production plans generated:

- satisfy the time, sequence and capacity constraints,

- optimise the use the capacities available,

- minimise the inventory costs, and

- are schedulable and realisable on the short-term.

The medium-term planning and the short-term, shop floor level scheduling are connected by aggregation (Váncza, et al., 2004). The latter - based on constraint programming (Kovács, et al., 2003) - takes the capacity constraints, the resource requirements of elementary operations, the precedence constraints between them, and the manufacturing, set-up and transfer times, into account.

\subsection{Simulation-supported production-scheduling}

The above system generates close-to-optimal production and capacity plans on the medium term, and detailed production schedules on the short term. Production plans and schedules, let them be generated by the most sophisticated methods, make not much sense if they cannot be executed. However, assumptions (e.g., concerning resource availability, production technology) taken by planning time are often violated at execution time. The closer we are to the realization of plans and schedules, the higher is the chance of unexpected events that can render plans and schedules inadequate. That is why practical scheduling is driven by uncertainty, and the methods applied in dynamic job shops rarely utilize theoretical results (McKay and Wiers, 1999).

However, the deterministic constraint-based factory model can hardly account for all the uncertain events, especially for those that may happen on the shop floor. Hence, factors are included into an appropriate simulation model which will be used for evaluating the results of the constraint-based scheduler in face of uncertainties.

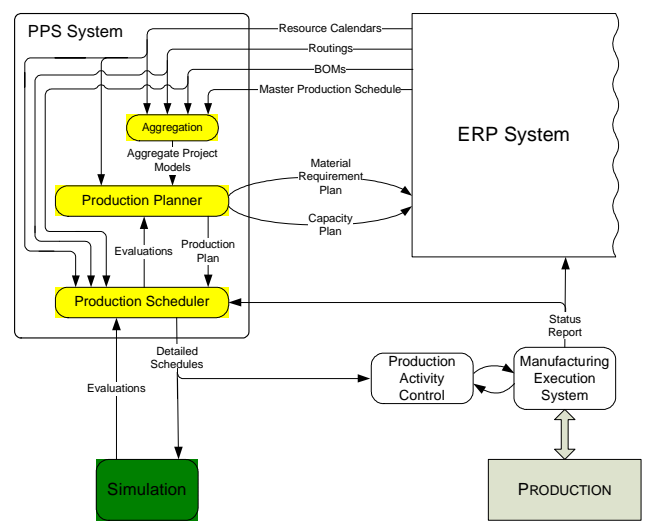

Fig. 4: Bridging the gap between ERP and MES

Uncertainties modelled within the simulation model relate to (1) delivery and (2) quality of incoming material; (3) machine downtimes, (4) processing times and (5) insertion of extra, adjustment operations into the routings (Kádár, et al., 2004,). It will offer a benchmark platform for the generated (close-to) optimal schedules, help to evaluate the robustness of daily schedules against the above uncertainties and to support the systematic test of the production planning and scheduling system (Fig. 4). From among the $R \& D$ results which led to prototypes tested and demonstrated in the industry and/or industrial applications let us outline the following - partly already mentioned - solutions:

- PROTERV: project-oriented capacity and production planner system,

- PROTERV-H: integrated production planner and scheduler system,

- IMUTA: interactive multimedia and tele-presence framework,

- INDOK: development framework for intelligent documents,

- M $^{2}$ ATRIX: monitoring-oriented simulator of production lines,

- PRODMOD: intelligent exploration of production dependencies.

\section{CONCEPT OF VITAL, THE NATIONAL PROJECT ON REAL-TIME, COOPERATIVE ENTERPRISES}

One of the most important trends in manufacturing is manifested in the paradigm of customised mass production, which means difficult to accomplish the task of producing customised products at a price near to the level in mass production. The national research project VITAL: Real-time Co-operative Enterprises IT-solutions for enterprises producing 
mass customized products and working in networks are to be developed. In addition to customised mass production, issues are to be handled, like

- globalisation, increasing competition, frequently changing, uncertain environment (Wiendahl, et al., 2002),

- growing complexity of production processes, manufacturing systems and enterprise structures,

- autonomous, partly competing, partly cooperating production structures.

Table 1 summarizes the challenges and the answers of VITAL.

\begin{tabular}{|l|l|}
\hline \multicolumn{1}{|c|}{ Challenges } & \multicolumn{1}{c|}{ VITAL's answers } \\
\hline Complexity & $\begin{array}{l}\text { Autonomy } \\
\text { cooperation }\end{array}$ \\
\hline $\begin{array}{l}\text { Changes and } \\
\text { disturbances }\end{array}$ & Intelligence \\
\hline $\begin{array}{l}\text { Finding the possible } \\
\text { best technical- } \\
\text { economic solutions }\end{array}$ & $\begin{array}{l}\text { Integration } \\
\text { optimisation }\end{array}$ \\
\hline Quick reaction ability & Real-timeness \\
\hline
\end{tabular}

The goal of the project is to research and develop new methods for the real-time management of complex technical and economic systems that work in changing, uncertain environments. Since the methods come from various, novel areas of informatics, operational research and knowledgebased systems, their integration will balance the aspects of optimisation, autonomy, and cooperation. Fig. 5 summarizes the main endeavours: the research and development of solutions from the level of production networks through single enterprises to production lines, which can ensure the optimal / near to optimal behaviour of the whole system, and moreover, in a real-time fashion required by the given level of production.

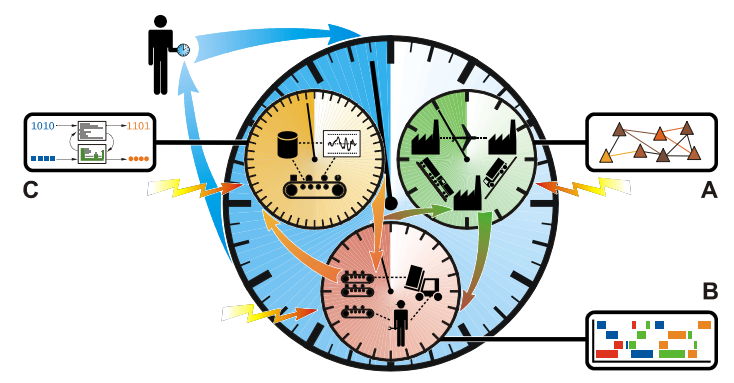

Fig. 5: General concept of the VITAL project.

The orders are to be fulfilled in good quality, on the agreed price and on time. The customers do not necessarily realize that they usually face a conglomerate of firms, i.e., production networks. The importance of the time is illustrated by the watches in the figure, which incorporates the different levels (network, enterprise, production line) of the production expected to react on the external and internal changes and disturbances (indicated by thunderbolts) with a reaction time characterising the level in question. The problems to be solved are as follows (referring to the notations of the Figure):
- integrated production planning and scheduling (B),

- real-time production control (C),

- management of distributed, cooperative systems (A).

The reason for the above sequence is that the highlevel resource-management and scheduling of enterprises can give the basis, on the one hand, for the reliable, optimal or near to optimal management of supply chains (Scholz-Reiter, et al., 2004) and production networks, and, on the other, for handling changes and disturbances in shop floors or production lines.

The following R\&D issues are addressed within the above enumerated clusters of the project:

\section{- Integrated production planning and scheduling:}

- development of novel mathematical models and related solution methods,

- combination of the methods of operations research (scheduling theory, linear and integer programming) and artificial intelligence (constraint programming),

- estimation of solutions' goodness,

- development of parametric, scalable modules for production optimisation,

- development of systems to be installed at the enterprises or for use as e-service.

\section{- Real-time production control:}

- modelling of disturbance and change sensitivities,

- - automatic situation recognition and related problem solving, decision support, machine learning,

- research on reactive and proactive rescheduling algorithms,

- development of reactive and proactive production control support systems,

- integration of active identifiers into production control.

- Management of distributed, cooperative systems:

- research on multi-agent systems,

- development of ontologies for exchanging production-related information,

- planning of negotiation mechanisms and communication protocols,

- development of models for describing production networks, behaviour analysis of the networks, development of efficient behaviour patterns.

\section{CONCLUSIONS}

The paper introduced the main $\mathrm{R} \& \mathrm{D}$ directions, i.e., adaptive manufacturing, digital manufacturing, knowledge-based manufacturing, and networked manufacturing, outlined in the European initiative Manufuture. However, these issues have to be handled in a holistic way, and at least two additional requirements, i.e., the real-timeness and cooperateveness of the whole system, have to be added, which are of high and increasing importance. The paper 
outlined the main results of a national $R \& D$ project in Hungary on Digital Enterprises, Production Networks. Decision support systems were developed within the project in order to help enterprises to cope with the problems of uncertainty and complexity, to increase their efficiency, to join in production networks and to improve the scope and quality of their customer relationship management.

The main concept of a new, just started, national research project VITAL: Real-time Cooperative Enterprises was also introduced. One of the most "vital" features of factories is their ability of cooperation, quick responses to changes and disturbances. These are matters of survival, independent of the size of the firms. The goal of the project is to research and develop new methods for the real-time management of complex technical and economic systems that work in changing, uncertain environments. Since the envisaged methods come from various, novel areas of informatics, operations research and knowledge-based systems, their integration is expected to balance the aspects of optimisation, autonomy, and cooperation.

\section{ACKNOWLEDGEMENTS}

The research was supported, partially, by the projects "Digital enterprises, production networks" and "Real-time, cooperative enterprises" in the frame of the National Research and Development Programme by the Ministry of Education, Hungary (Grant Nos. 2/040/2001 and 2/010/2004). A part of the work was covered by the National Research Fund, Hungary, Grant Nos. T043547, T046509 and T049486. Botond Kádár greatly acknowledges the support of Bolyai János Scholarship.

\section{REFERENCES}

Wiendahl, H.-P., Scholtissek, P. (1994). Management and control of complexity in manufacturing, Annals of the CIRP, 43/2: 533-540.

Wiendahl, H.-P., Lutz, S. (2002). Production in Networks, Annals of the CIRP, 51/2: 1-14.

Maropoulos, P.G. (2002). Digital Enterprise Technology - Defining Perspectives and Research Priorities, Proc. of the $1^{\text {st }}$ CIRP (UK) Sem. on Digital Enterprise Technology, DET02, September 16-17, Durham, UK, Part V: 3-12.

Monostori, L.; Váncza, J.; Márkus, A.; Kádár, B.; Viharos Zs.J. (2003). Towards the Realization of Digital Enterprises, $36^{\text {th }}$ CIRP Int. Seminar on Manufacturing Systems, June 3-5, Saarbrücken, Germany: 99-106.

Monostori, L., Márkus, A., Van Brussel, H., Westkämper, E. (1996). Machine learning approaches to manufacturing, Annals of the CIRP, 45/2: 675-712.

Monostori, L. (2003) AI and machine learning techniques for managing complexity, changes and uncertainties in manufacturing, Engineering Applications of Artificial Intellig., 16/4: 277-291.
N.N. (2003) Working Document for the Manufuture 2003 Conference, Manufuture 2003: European Manufacturing of the Future: Role of Research and Education for European Leadership, Dec. 12, 2003, Milano, Italy: 1-51.

N.N. (2004). Manufuture: A Vision for 2020, Assuring the Future of Manufacturing in Europe, EC, November: 1-20.

Hatvany, J. (1983). The efficient use of deficient information, Annals of the CIRP, 32/1: 423-425.

Ueda, K., Vaario, J., Fujii, N. (1998). Interactive Manufacturing: Human Aspects for Biological Manufacturing Systems, Annals of CIRP 47/1: 389-392.

Ueda, K.; Márkus, A.; Monostori, L.; Kals, H.J.J.; Arai, T. (2001): Emergent synthesis methodologies for manufacturing, Annals of the CIRP, 50/2: 535-551.

Teti, R., Kumara, S.R.T. (1997). Intelligent Computing Methods for Manufacturing Systems, Annals of the CIRP, 46/2: 629-652.

Monostori, L., Haidegger, G., Váncza, J., Viharos, Zs.J. (2002). Digital Enterprises: A national R\&D project in Hungary, Proc. of the $1^{\text {st }}$ CIRP Sem. on Digital Enterprise Technology, DET02, September 16-17, Durham, UK, 269-272.

Váncza, J.; Kis, T.; Kovács, A. (2004). Aggregation The Key to Integrating Production Planning and Scheduling, Annals of the CIRP 53/1: 377-380.

Márkus, A.; Váncza, J.; Kis, T.; Kovács, A. (2003). Project Scheduling Approach to Production Planning, Annals of the CIRP, 52/1: 359-362.

Kovács, A., Váncza, J., Kádár, B., Monostori L., Pfeiffer, A. (2003). Real life scheduling using constraint programming and simulation, Proc. of IMS 2003, $7^{\text {th }}$ IFAC Workshop on IMSs, Budapest, Hungary: 233-239.

Kovács, A.; Márkus, A.; Váncza, J. (2004). Structural Exploration of Constraint-Based Scheduling Problems, Proc. of the $37^{\text {th }}$ CIRP Int. Seminar on Manufacturing Systems; May 19-21, Budapest, Hungary: 433-439.

Kis, T. (2005). A branch-and-cut algorithm for scheduling projects with variable-intensity activities, Mathematical Programming, 103/3: 515-539.

McKay, K.N., Wiers, V.C.S. (1999). Unifying the Theory and Practice of Production Scheduling, $J$. of Manufacturing Systems, 18/4: 241-255.

Kádár, B., Pfeiffer, A., Monostori, L. (2004). Discrete event simulation for supporting production planning and scheduling decisions in digital factories, Proc. of the $37^{\text {th }}$ CIRP Int. Seminar on Manufacturing Systems; May 19-21, Budapest, Hungary: 441-448.

Wiendahl, H.-H., Roth, N., Westkämper, E. (2002). Logistical Positioning in a Turbulent Environment, Annals of the CIRP, 51/1: 383-386.

Scholz-Reiter, B., Höhns, H., Hamann, T. (2004). Adaptive Control of Supply Chains: Building Blocks and Tools of an Agent-Based Simulation Framework, Annals of the CIRP, 53/1: 353-356. 